\title{
A Rare Case of an Adult Congenital Diaphragmatic Hernia be- coming Symptomatic after an Elective Procedure
}

\author{
Stylianos Voulgarelis ${ }^{1^{*}}$ and Sylvia Y Dolinski ${ }^{2}$ \\ ${ }^{1}$ Divisions of Pediatric and Adult Cardiac Anesthesiology, Medical College of Wisconsin, USA \\ ${ }^{2}$ Department of Anesthesiology, Medical College of Wisconsin, USA
}

*Corresponding author: Stylianos Voulgarelis, M.D., Assistant Professor of Anesthesiology, Divisions of Pediatric and Adult Cardiothoracic Anesthesiology, Medical College of Wisconsin, USA, Tel: (414)266-5910 (Children's Hospital of Wisconsin)

\begin{abstract}
Summary
Congenital diaphragmatic hernias may run undiagnosed for years before a change in symptomatology may lead to the diagnosis and treatment. We present a 63-year-old previously asymptomatic patient that developed progressive symptomatology after a laparoscopic procedure. The increased intraabdominal pressure related to the peritoneal insufflation and Trendelenburg intraoperative positioning in combination with the failure of the surgeon to perform the initial laparoscopic exploration of the whole abdomen resulted in an increased amount of viscera herniated through the diaphragm. The weeks following his elective surgery he presented multiple time to the emergency department before the correct diagnosis was made and his diaphragmatic defect gets repaired.
\end{abstract}

\section{Introduction}

Hiatal hernias are very common in adults. Sliding hernias consist the $95 \%$ of them and present with epigastric pain, retrosternal tightness or heartburn. They rest are paraesophageal hernias with part of the stomach being herniated through the esophageal hiatus or a combination of them. When part of the stomach is herniated through the diaphragm the risk of strangulation of obstruction is very high. All these are acquired hernias and most of the time they develop due to increased intraabdominal pressure (obesity, chronic cough). Rarely, adults present with congenital diaphragmatic defects that run undiagnosed for decades before they give symptoms, either non-specific (epigastric pain, dyspnea) or catastrophic (colon strangulation).

\section{Report}

A 63-year-old male with a history of GERD, and chronic constipation presented for an elective laparoscopic bilateral inguinal and ventral hernia repairs. He had intraoperative issues with $V / Q$ mismatch and an obstructive pattern on his end-tidal $\mathrm{CO}_{2}$ tracing. The elective procedure was concluded with him treated intraoperatively with albuterol and maintained on $\mathrm{FiO}_{2}$ 1.0. At the end of the procedure he was extubated on 4 $\mathrm{L} /$ min oxygen by nasal cannula (NC). His postoperative CXR Showed opacification in the mid and lower left lung and subcutaneous emphysema in the chest wall, presumably from recent laparoscopic surgery (Figure 1).

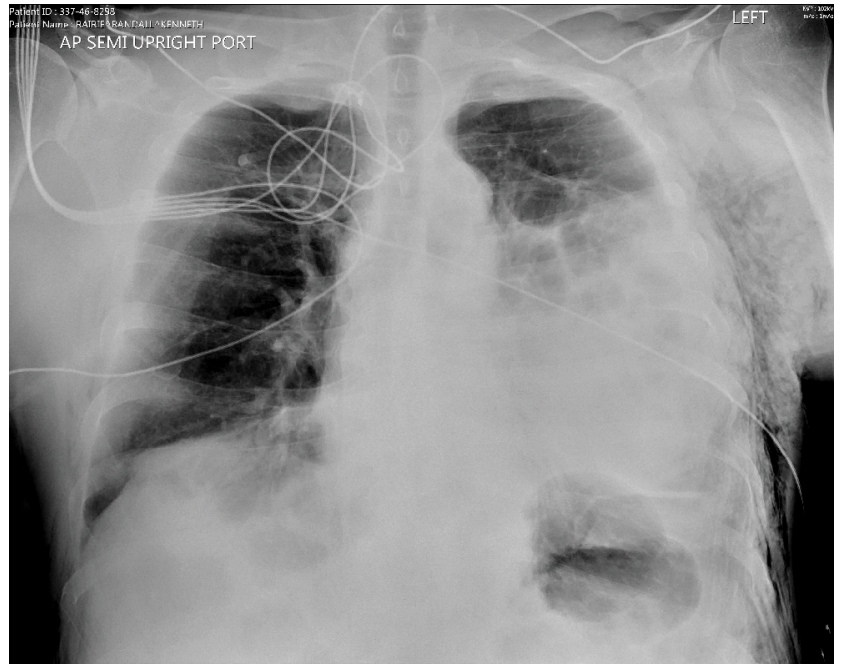

Figure 1: Anteroposterior chest radiograph obtained in the recovery room showing a left mid and lower lung opacity.

Citation: Voulgarelis S, DolinskiSY (2018)A Rare Case of an Adult Congenital Diaphragmatic Hernia becoming Symptomatic after an Elective Procedure. Int J Anesthetic Anesthesiol 5:078. doi.org/10.23937/23774630/1410078 


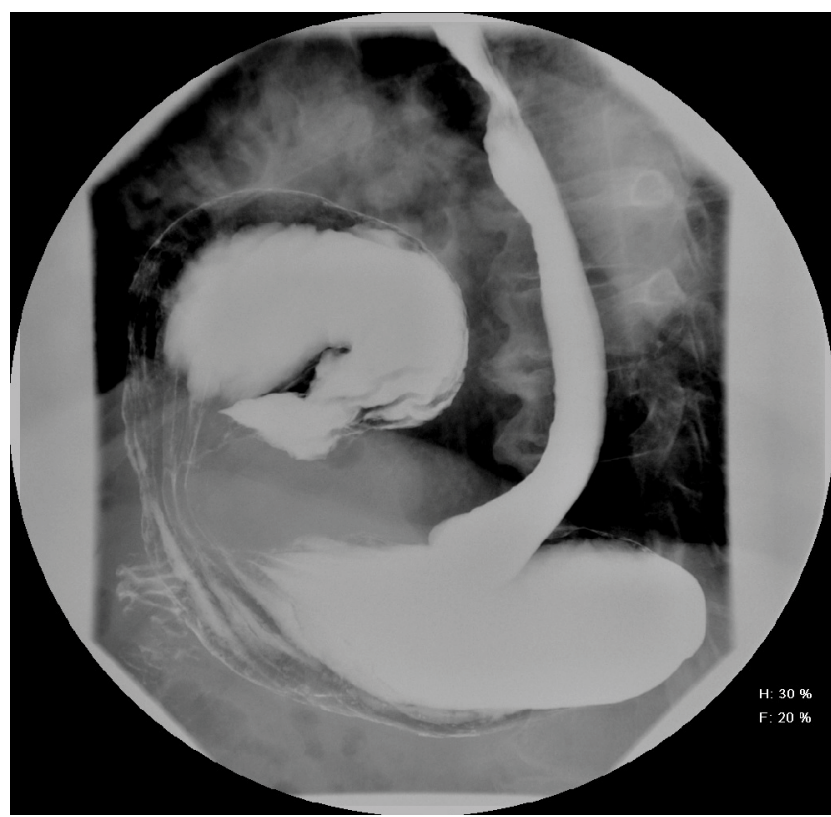

Figure 2: Upper GI study with PO barium showing herniation of the antrum in the chest.
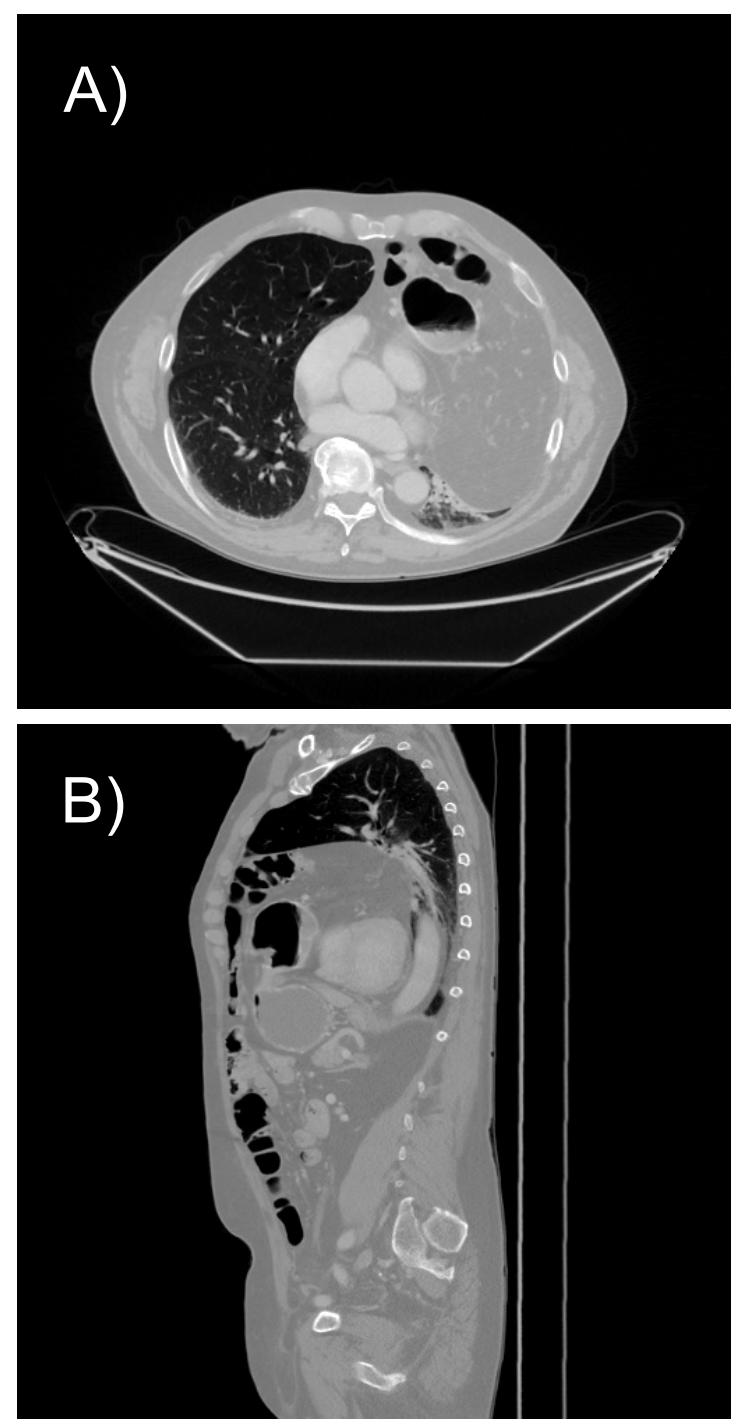

Figure 3: a) CT scan with IV contrast showing bowel loops and the spleen in the left hemithorax; b) CT scan with IV contrast showing substernal herniation of colon in the thorax. Herniation of half the stomach and spleen in the left thorax. no evidence of bowel obstruction.
The Patient was admitted for a 23 hour observation. His oxygenation improved over night and was weaned to room air. He was discharged home after tolerating a diet. The following three weeks he complained of non-specific symptoms of vomiting/constipation, belching and not feeling well. He visited the emergency department and underwent a barium upper Gl study that revealed mesoaxial gastric volvulus within a left diaphragmatic hernia and spontaneous gastric reflux (Figure 2).

He underwent a upper GI endoscopy in an attempt to reduce the volvulus which was unsuccessful.

His symptoms persisted and included brown ground emesis. He underwent a CT with contrast that showed a large anterior diaphragmatic hernia with herniation of the distal stomach, proximal duodenum, and a portion of the transverse colon without evidence of bowel obstruction (Figure $3 a$ and Figure $3 b$ ).

He was scheduled for a laparoscopic repair of a Morgagni hernia. (Figure $4 a$, Figure $4 b$ and Figure $4 c$ ) during

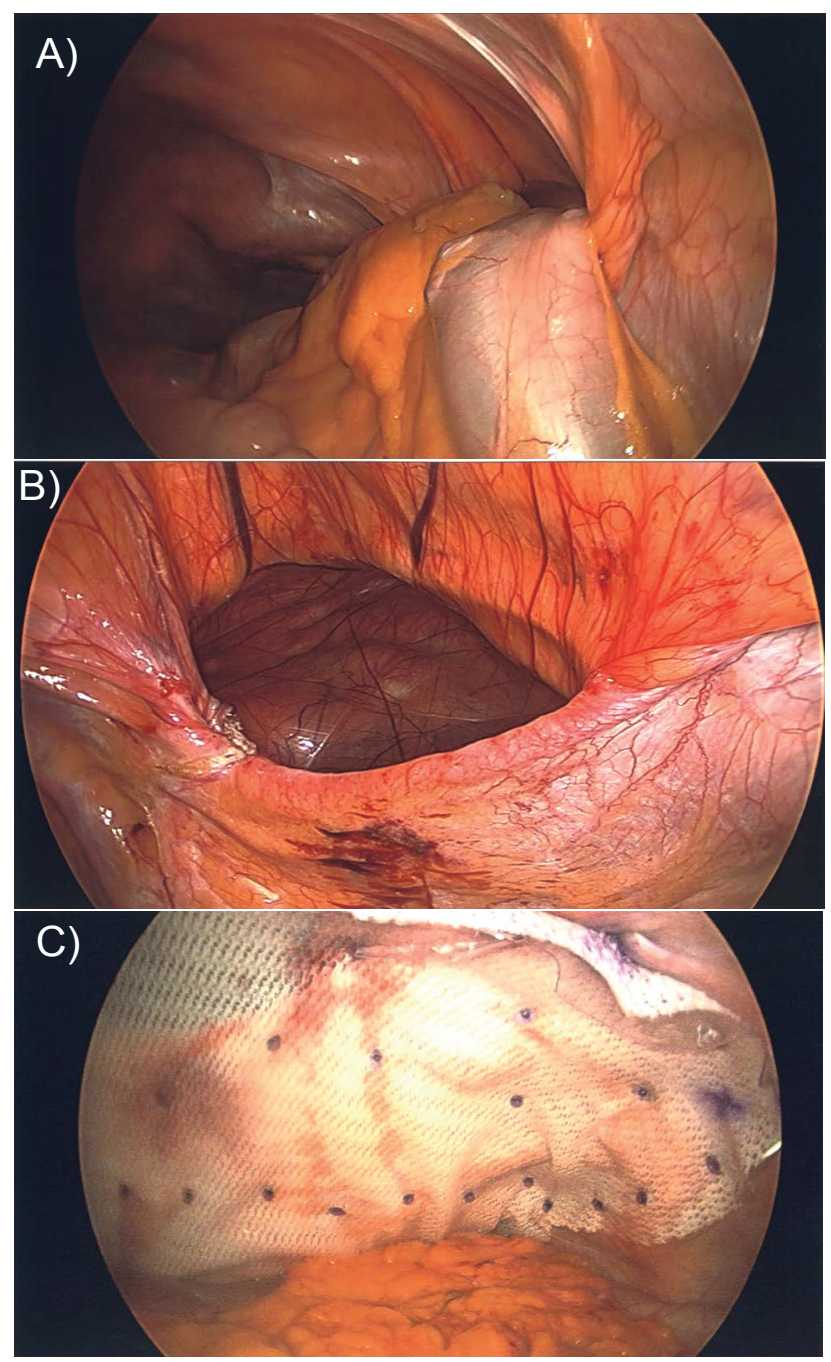

Figure 4: Intraoperative pictures. a) Showing herniation of the peritoneal contents through the Morgagni hernia; $b$ ) Showing the diaphragmatic defect after reduction of the herniated contents back in the peritoneal cavity. The pericardium is seen in the defect; c) The defect has been repaired by a laparoscopic mesh placement. 


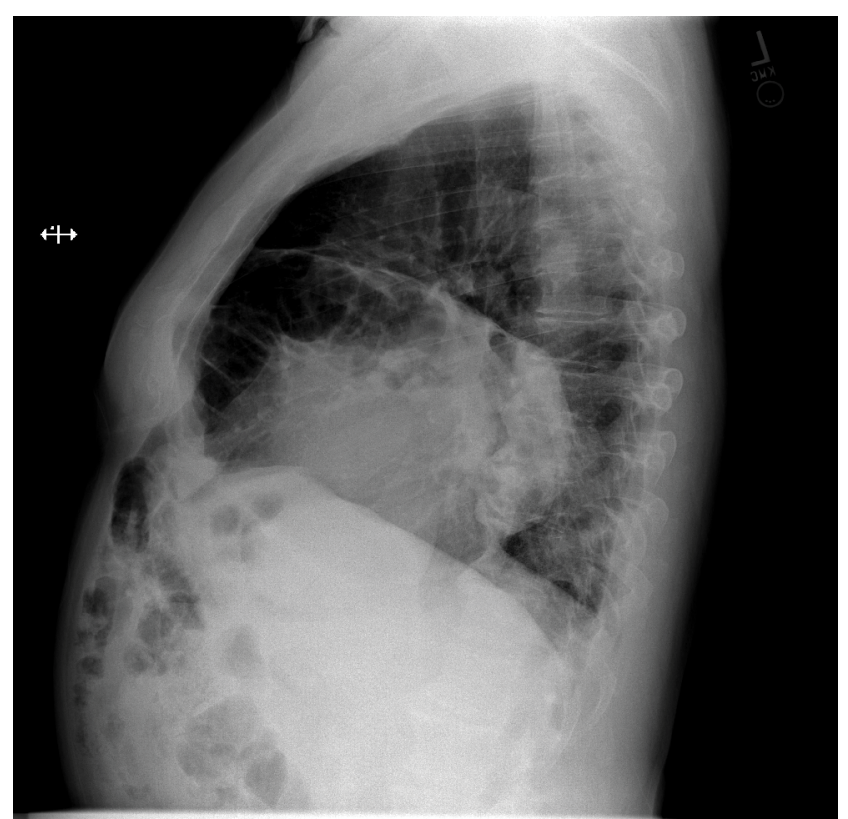

Figure 5: Lateral chest radiograph showing large bowel loop posteriorly to the sternum.

any laparoscopic procedure, a general visualization of the abdominal cavity must be performed.

If the surgeon had performed one during the first procedure the Morgagni hernia would have been discovered and the diagnostic process would have been shortened significantly.

\section{Discussion}

Congenital diaphragmatic hernias have a prevalence of 1 every 2000-4000 live births. It consists of either a defect or thinning and incomplete muscularization (eventration) of the diaphragm. The defect may be posterolateral (Bochdalek), retrosternal (Morgagni or pentalogy of Cantrell) or central.

Eighty to nighty percent of all congenital diaphragmatic defects are left sided (Bochdalek hernias). The diagnosis and risk stratification (side of hernia, concomitant heart defects, lung to thorax ratio) can be made prenatally. The delivery is planned at a specialized center as these patients often present with severe respiratory symptoms. The treatment is focused on the support of the severely underdeveloped lung and the stabilization of pulmonary hypertension. The repair can be performed in an open fashion through an abdominal approach or thoracoscopically. Additionally, care may involve oscillator or ECMO support.
On the other hand, the more frequently right-sided hernia of Morgagni ( $2 \%$ of all CDH cases) presents rarely with respiratory distress in infancy. The pediatric patient will exhibit frequent episodes of respiratory infections or bowel obstruction. It is not uncommon that a hernia of Morgagni remains undiagnosed until adulthood at which time it presents with bowel obstruction or respiratory symptoms as in this case. A hiatal hernia can be differentiated from a hernia of Morgagni by its symptoms of epigastric pain or acid reflux.

In adults the defect grows slowly over many years and may remain totally asymptomatic. This is a rare but potentially fatal disease especially if it involves strangulation of part of the colon.

However it may also present with nonspecific abdominal pain, soft abdomen and shortness of breath. A chest $X$-ray may be misleading. A lateral chest X-ray (Figure 5) and a barium enema X-ray or CT aid in revealing the diagnosis.

\section{Conclusions}

An initial laparoscopic exploration of the whole abdominal cavity is mandatory in any laparoscopic procedure. Even minor laparoscopic procedures may unmask, due to insufflation previously asymptomatic congenital diaphragmatic defects [1-5].

\section{Acknowledgments}

No external funding or competing interests declared.

\section{References}

1. S Arora, A Haji, P Ng (2008) Adult morgagni hernia: The need for clinical awareness, early diagnosis and prompt surgical intervention. Ann R Coll Surg Engl 90: 694-695.

2. Enrica Bianchi, Paola Mancini, Stefania De Vito, Elena Pompili, Samanta Taurone, et al. (2013) Congenital asymptomatic diaphragmatic hernias in adults: A case series. Journal of Medical Case Reports 7: 125.

3. Adrian T Bosenberg, Robin A Brown (2008) Management of congenital diaphragmatic hernia. Current Opinion in Anaesthesiology 21: 323-331.

4. TPF Loong, HM Kocher (2005) Clinical presentation and operative repair of hernia of Morgagni. Postgrad Med J 81: $41-44$

5. Akinkuotu AC, Cruz SM, Abbas PI, Lee TC, Welty SE, et al. (2016) Risk-stratification of severity for infants with $\mathrm{CDH}$ : Prenatal versus postnatal predictors of outcome. J Pediatr Surg 51: 44-48. 